\title{
Development and Implementation of Non-Newtonian Rheology Into the Generalized Fluid System Simulation Program (GFSSP)
}

\author{
Roberto Di Salvo', Stelu Deaconu, ${ }^{2}$, and Alok Majumdar ${ }^{3}$ \\ ${ }^{1,2}$ CFD Research Corporation \\ 215 Wynn Dr. Huntsville, Alabama 35805 \\ ${ }^{3}$ ER43/Thermal \& Combustion Analysis Branch \\ NASA/Marshall Space Flight Center \\ Huntsville, Alabama 35812
}

One of the goals of this program was to develop the experimental and analytical/computational tools required to predict the flow of non-Newtonian fluids through the various system components of a propulsion system: pipes, valves, pumps etc. To achieve this goal we selected to augment the capabilities of NASA's Generalized Fluid System Simulation Program (GFSSP) software. GFSSP is a generalpurpose computer program designed to calculate steady state and transient pressure and flow distributions in a complex fluid network. While the current version of the GFSSP code is able to handle various systems components the implicit assumption in the code is that the fluids in the system are Newtonian. To extend the capability of the code to non-Newtonian fluids, such as silica gelled fuels and oxidizers, modifications to the momentum equations of the code have been performed. We have successfully implemented in GFSSP flow equations for fluids with power law behavior.

The implementation of the power law fluid behavior into the GFSSP code depends on knowledge of the two fluid coefficients, $n$ and $K$. The determination of these parameters for the silica gels used in this program was performed experimentally. The $n$ and $K$ parameters for silica water gels were determined experimentally at CFDRC's Special Projects Laboratory, with a constant shear rate capillary viscometer.

Batches of 8:1 (by weight) water-silica gel were mixed using CFDRC's 10-gallon gelled propellant mixer. Prior to testing the gel was allowed to rest in the rheometer tank for at least twelve hours to ensure that the delicate structure of the gel had sufficient time to reform. During the tests silica gel was pressure fed and discharged through stainless steel pipes ranging from 1" to 36 " in length and three diameters; $0.023 ", 0.032 \%$, and 0.047 '. The data collected in these tests included pressure at tube entrance and volumetric flowrate. From these data the uncorrected shear rate, shear stress, residence time, and viscosity were evaluated using formulae for non-Newtonian, power law fluids. The maximum shear rates (corrected for entrance effects) obtained in the rheometer with the current setup were in the 150,000 to $170,000 \mathrm{sec}^{-1}$ range.

GFSSP simulations were performed with a flow circuit simulating the capillary rheometer and using Power Law gel viscosity coefficients from the experimental data. The agreement between the experimental data and the simulated flow curves was within $\pm 4 \%$ given quality entrance effect data.

\footnotetext{
${ }^{1}$ SRTC/ Senior Member AIAA

${ }^{2}$ Member AIAA

${ }^{3}$ Senior Member AIAA
} 


\section{Introduction}

The goal was to develop the experimental and analytical/computational tools required to predict the flow of non-Newtonian fluids through the various system components of a propulsion system: pipes, valves, pumps etc. To achieve this objective, we selected to augment the capabilities of NASA's Generalized Fluid System Simulation Program (GFSSP) software. GFSSP is a generalpurpose computer program designed to calculate steady-state and unsteady-state pressure and flow distributions in a complex fluid network. While the current version of the GFSSP code is able to handle various systems components, the implicit assumption in the code is that the fluid in the system is Newtonian. To extend the capability of the code to non-Newtonian fluids, such as silica gelled fuels and oxidizers, modifications to the momentum equations of the code were performed.

We begin by examining the discretized momentum conservation equation shown below in Eq. 1 .

$$
\begin{aligned}
& \frac{\left(m_{i j} u_{i j}\right)_{\tau+\Delta \tau}-\left(m_{i j} u_{i j}\right)_{\tau}}{\Delta \tau}+\frac{\dot{m}_{i j}}{g_{c}}\left(u_{i j}-u_{u}\right)= \\
& \left(p_{i}-p_{j}\right) \mathrm{A}_{\mathrm{ij}}+\frac{\rho_{u} g V_{i j} \cos \theta}{g_{c}}-K_{f} \dot{m}_{i j}\left|\dot{m}_{i j}\right| \mathrm{A}_{\mathrm{ij}} \\
& +\frac{\rho_{u} K_{r o t}^{2} \omega^{2}}{2 g_{c}} \mathrm{~A}_{i j}\left(r_{j}^{2}-r_{i}^{2}\right)+S_{i j}
\end{aligned}
$$

Eq. 1

This equation represents the balance of fluid forces acting on a given branch [Van Hooser, 1999]. A typical branch configuration is shown below in Figure 1. As outlined in Van Hooser [1999], the two terms on the left side of Eq. 1 represent the inertia of the fluid. The first term denotes the time dependence and must be considered for unsteady calculations. The second term is significant when there is a large change in area or density from branch to branch. The first term on the right side of the momentum equation represents the pressure gradient in the branch. The second term represents the effect of gravity. The gravity vector makes an angle $(\theta)$ with the assumed flow direction vector. The third term represents the frictional effect. Friction is modeled as a product of $\mathrm{K}_{\mathrm{f}}$, the square of the flow rate, and area. $K_{f}$ is a function of the fluid density in the branch and the nature of flow passage being modeled by the branch. The fourth term in the momentum equation represents the effect of the centrifugal force. This term will be present only when the branch is rotating as shown in Figure 1. $\mathrm{K}_{\text {rot }}$ in this term is a factor representing fluid rotation and is unity when the fluid and the surrounding solid surface rotate at the same speed. This term also requires the radial distances from the upstream and downstream faces of the branch to the axis of rotation.

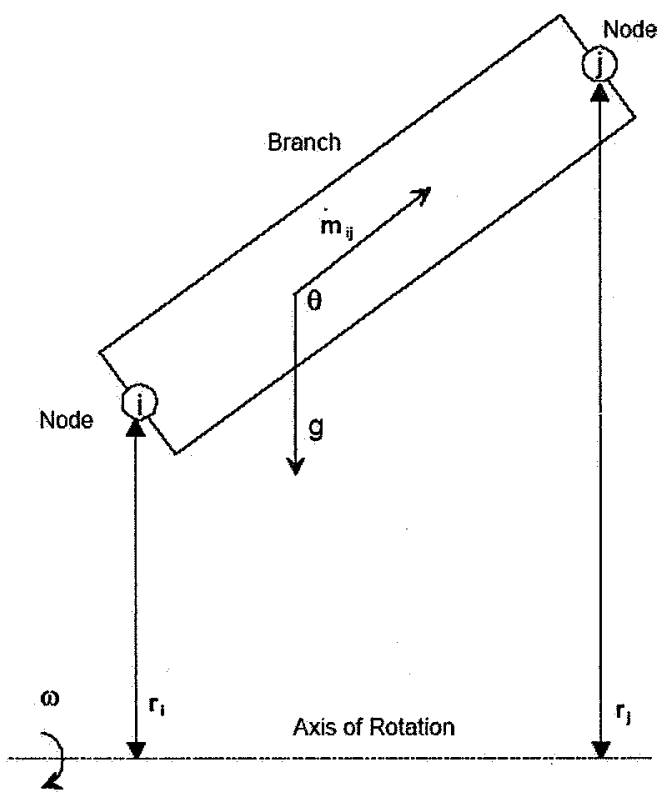

Figure 1 - Schematic of a GFSSP Flow Branch Showing Sources of Momentum (from Van Hooser, 1999)

To account for non-Newtonian behavior, such as power law, the third term (friction effect) on the right side of the equation must be modified. The modification to the code is performed in a user subroutine, SORCEF, which is compiled and added to the GFSSP executable core at runtime. Figure 2 illustrates the interaction of the SORCEF user subroutine with the GFSSP solver module [Majumdar, 1999]. 
Solver Modulelimettion User Subroutinelinedion

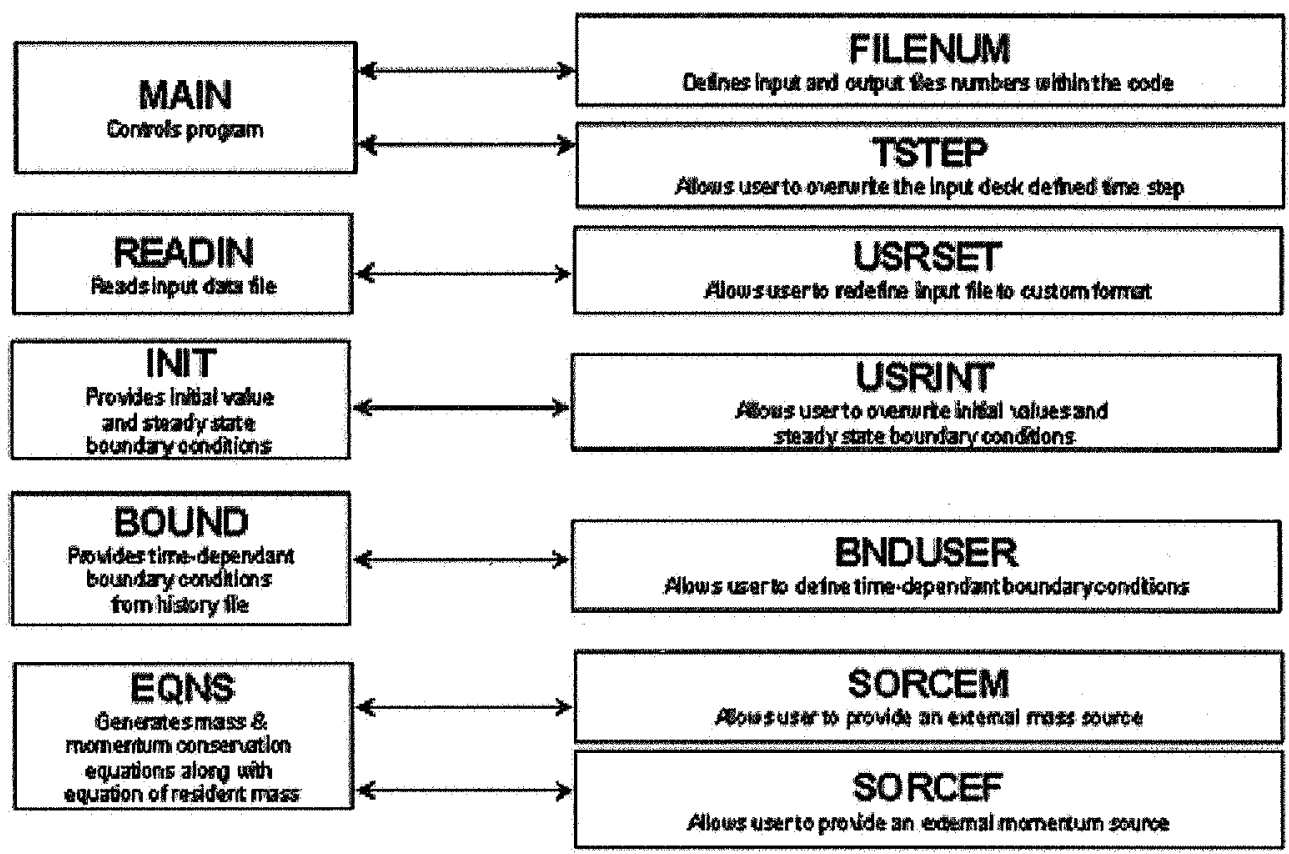

Figure 2 - Interaction of User Subroutine with Solver Module (from Majumdar, 1999)

The modifications to the friction momentum term (TERM4) in SORCEF consist of the calculation of a new shear rate for power law fluids and the relative friction force,

$$
\begin{aligned}
& \dot{\gamma}=\left(\frac{3 n+1}{n}\right) \cdot\left(\frac{Q}{\pi \cdot r^{3}}\right), \\
& F_{f}=\tau_{f} \cdot A_{l}=K \cdot \dot{\gamma}^{n} \cdot\left(2 \pi \cdot r \cdot L_{b}\right)
\end{aligned}
$$

where $n$ is the flow behavioral index, $K$ is the consistency of the fluid, and $\mathrm{r}$ is the radius of the cross section of the flow branch under consideration. The implementation of the power law fluid behavior into the GFSSP code depends on knowledge of the two fluid coefficients, $n$ and $K$. The determination of these parameters for the silica gels used in this program must be performed experimentally. The $n$ and $K$ parameters for silica water gels were determined experimentally at CFDRC's Special Projects Laboratory using a capillary rheometer. The controller on the rheometer can be programmed to run in either controlled shear rate (constant flow-rate) or controlled shear stress (constant pressure) modes. The tests run with the silica gels were performed in constant shear rate mode. Data acquisition was performed with LabVIEW.

\section{Silica Gel Testing}

The batch of silica gel used in all the tests was mixed with the CFDRC 10-gallon gel mixer. Prior to testing the gel was allowed to rest in the rheometer tank for at least twelve hours to ensure that the delicate structure of the gel had sufficient time to reform. During the tests silica gel was pressure fed and discharged through stainless steel pipes of 1-36in length and three diameters 0.023 , 0.032 , and $0.047 \mathrm{in}$. The capillary tubes were soldered to 1/4in nipples to be used with standard high-pressure fittings, and the tubes protruded out of the nipple $3 / 8$ to $1 / 2 \mathrm{in}$. This resulted into a reentrant flow condition at the entrance of the capillary and an entrance pressure loss that needed to be evaluated experimentally. The pressure transducers were calibrated to within $\pm 1 \%$ of reading by means of an Omega dead-weight tester. The flowrate was measured with an in-line McMillan turbine flowmeter. The accuracy of the flowmeter was checked by recording the time needed to fill calibrated laboratory beakers, and was found to be better than $\pm 2 \%$. The 


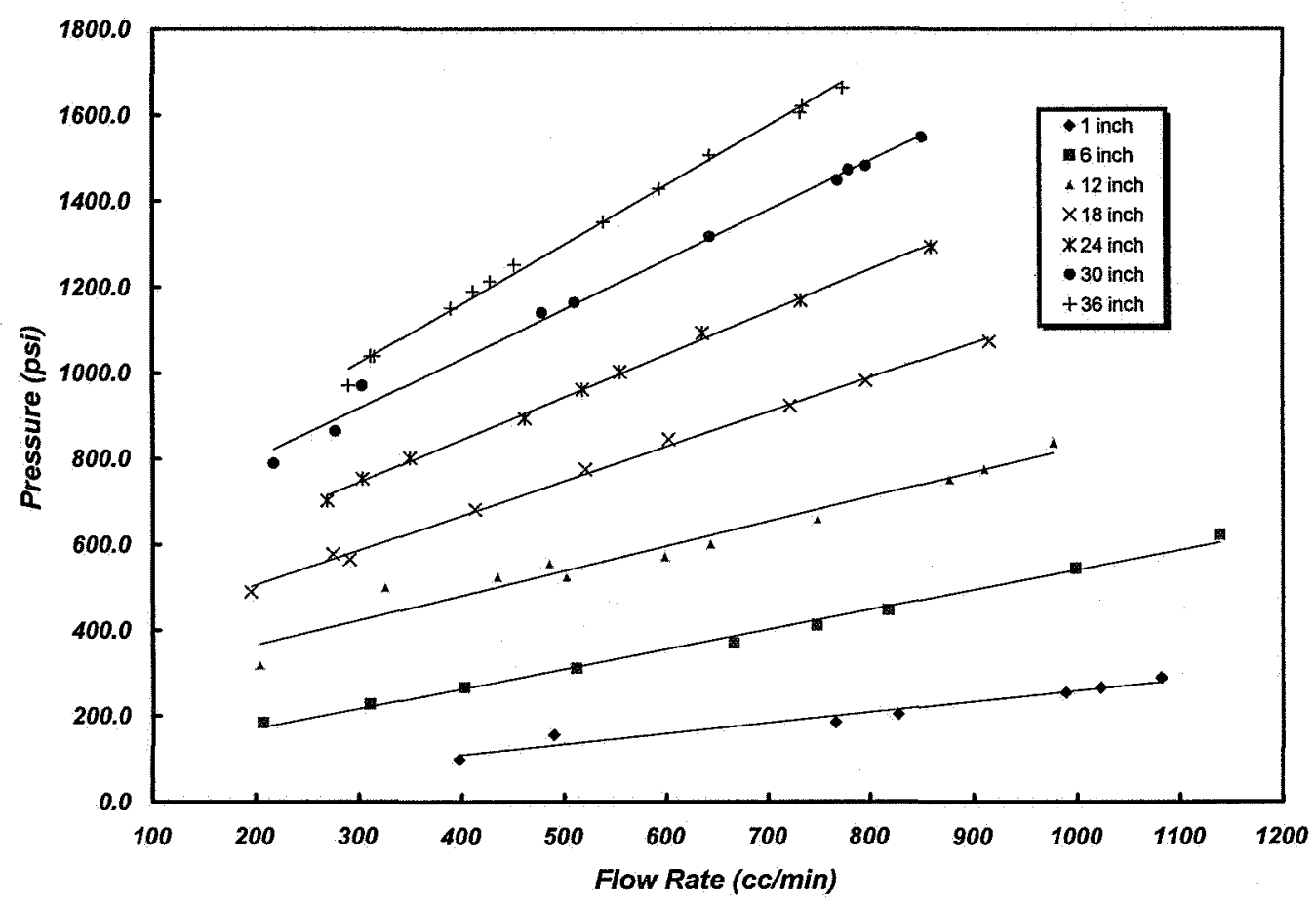

Figure 3 - Pressure Drop Versus Flowrate for 0.032in Tube of 1-36in Length. Pressure Drop Includes Entrance Pressure Loss

temperature of the gel during testing was monitored with an Omega DP-80 digital thermometer and was $21^{\circ} \mathrm{C} \pm 1{ }^{\circ} \mathrm{C}$. The data collected in these tests were: pressure at tube entrance and volumetric flowrate. From these the uncorrected shear rate, shear stress, residence time, and viscosity were evaluated using formulae for non-Newtonian power law fluids.

\begin{tabular}{ccc}
\hline $\begin{array}{c}\text { Tube } \\
\text { Diameter } \\
\text { [in] }\end{array}$ & $\begin{array}{c}\text { Length } \\
\text { [in] }\end{array}$ & Day \# \\
\hline 0.023 & 34 & 1 \\
0.032 & 36 & 1 \\
0.032 & 36 & 4 \\
0.047 & 36 & 1 (Test 1) \\
0.047 & 36 & 1 (Test 2) \\
0.047 & 36 & 4 (Test 1) \\
0.047 & 36 & 4 (Test 2) \\
0.032 & 30 & 5 \\
0.032 & 24 & 5 \\
0.032 & 18 & 5 \\
0.032 & 12 & 5 \\
0.032 & 6 & 5 \\
0.032 & 1 & 5 \\
\hline \hline
\end{tabular}

Table 1 - Summary of Tests for Gelled Silica
The maximum shear rates (corrected for entrance effects) obtained in the rheometer with the current setup were in the $150,000-170,000 \mathrm{sec}^{-1}$.

Table 1 lists the tests performed, the diameter /length of the stainless steel capillary tubes used, and the time passed between the gel mixing and the test time (in days). The tests were run over a one-week period in order to evaluate what the effect of gel settle-time is on the rheological properties of the gel. The $0.032 \mathrm{in}$ tube was cut in 6 in decrements and tested successively in day five in order to infer the entrance effects on gel flow.

\section{Analysis of the Gel Data}

In this section we present a preliminary analysis that has been performed on the gel data collected. The 0.032in tube data yielded the entrance loss coefficients for the reentrant flow. Figure 4.27 is a plot of the data collected in the six tests of day five and one test (36in) of day four.

The entrance effect pressure drop was estimated according to the Couette method, as outlined in Ferguson and Kemblowski (1991). The pressure 
drop per unit length of pipe according to this method is

$\frac{\Delta P}{\Delta L}=\frac{\Delta P_{2}-\Delta P_{1}}{L_{2}-L_{1}}$

Eq. 3

where $\Delta \mathrm{P}_{2} \Delta \mathrm{P}_{1}$, are measured pressure drops at the same flowrate, and $L_{2}, L_{1}$ are the lengths of the tube tested. The method works best if the tubes are much longer than the entrance effect length. Thus, considering the data from the 30 in and 24 in tests, the pressure drop per unit length of pipe was $\Delta \mathrm{P} / \Delta \mathrm{L}=38 \mathrm{psi} / \mathrm{in}$. The pressure drop per unit length of pipe is varying slightly across the measured flowrate and tube length range, but for simplicity the $38 \mathrm{psi} / \mathrm{in}$ value will be used to estimate the pressure loss due to reentrant flow at tube entrance.

Choosing from the middle of the flowrate/length range, $600 \mathrm{~mL} / \mathrm{min}$ and $18 \mathrm{in}$, the estimated pressure loss due to entrance effects is,

$\Delta \mathrm{P}_{\mathrm{e}}=\Delta \mathrm{P}_{\text {tot }}-(\Delta \mathrm{P} / \Delta \mathrm{L}) \cdot \mathrm{L}=824 \mathrm{psi}-38 \mathrm{psi} / \mathrm{in} \cdot 18 \mathrm{in}$ $=140 \mathrm{psi}$

It is useful to define the pressure loss effect in terms of the dynamic pressure in the tube [Munson, 1990].

$$
K_{e}=\frac{\Delta P_{e}}{\frac{1}{2} \rho V^{2}}
$$

Eq. 4

For the 18 in tube flowing $600 \mathrm{~mL} / \mathrm{min}$ the average velocity is $\mathrm{V}=19.4 \mathrm{~m} / \mathrm{sec}$, and the dynamic pressure is $27.12 \mathrm{psi}$. The entrance loss coefficient is $K_{\mathrm{e}}=5.16$. We note that the pressure loss due to entrance effect is five times greater than the dynamic pressure in the tube. In comparison, the reentrant loss coefficient for water flow [Munson, 1990] is only $K_{e \text { water }}=0.8$. Generally, the entrance loss coefficient is dependent on geometry and Reynolds number. However, in most cases of interest the loss coefficient is only a function of geometry. For now we will assume a constant entrance loss coefficient for all tubes of $K_{\mathrm{e}} \cong 5$ (It will be shown in the next section that this assumption introduces some error in the determination of the flow-curves for different pipe diameters). Using this value and the average velocity, the corrected pressure drop along the tubes can be calculated.

The non-Newtonian, power law fluid shear stress and shear rate are described by the following equations,

$$
\begin{aligned}
& \tau=\frac{d \cdot \Delta P}{4 \cdot L}, \\
& \Delta P=\frac{4 L \cdot k}{d}\left(\frac{8 Q}{\pi \cdot d^{3}}\right)^{n}\left(\frac{3 n+1}{n}\right)^{n}, \quad \text { Eq. } 5 \\
& \dot{\gamma}=\left(\frac{3 n+1}{n}\right) \frac{8 Q}{\pi \cdot d^{3}}
\end{aligned}
$$

where $L$ is the length of the tube, $d$ is the diameter, $k$ is a consistency factor $\left(\mathrm{Pa} \cdot \mathrm{Sec}^{\mathrm{n}}\right)$, and $\mathrm{n}$ is the behavioral index. The flow curve of a power law, time independent fluid is

$$
\tau=k \cdot \dot{\gamma}^{n}
$$

Eq. 6

The determination of $\mathrm{k}$ and $\mathrm{n}$ can be done by taking advantage of the quasi-linear behavior of the data in log-log representation. Replacing the shear stress and shear rate with the values in Eq. 5, the shear stress becomes,

$$
\begin{aligned}
& \ln \left(\frac{d \cdot \Delta P}{4 L}\right)=\ln (k)+n \cdot \ln \left(\frac{3 \cdot n+1}{4 \cdot n}\right), \quad \text { Eq.7 } \\
& +n \cdot \ln \left(\frac{8 \cdot \mathrm{v}}{\mathrm{d}}\right)
\end{aligned}
$$

We solve for $\mathrm{k}$ and $\mathrm{n}$ by fitting Eq. 7 to the data and obtain $\mathrm{n}=0.349$ and $\mathrm{k}=23.06 \mathrm{~Pa} \cdot \mathrm{sec}^{\mathrm{n}}$. The quasi-linear behavior of the data scaled logarithmically is evident in Figure 4. 


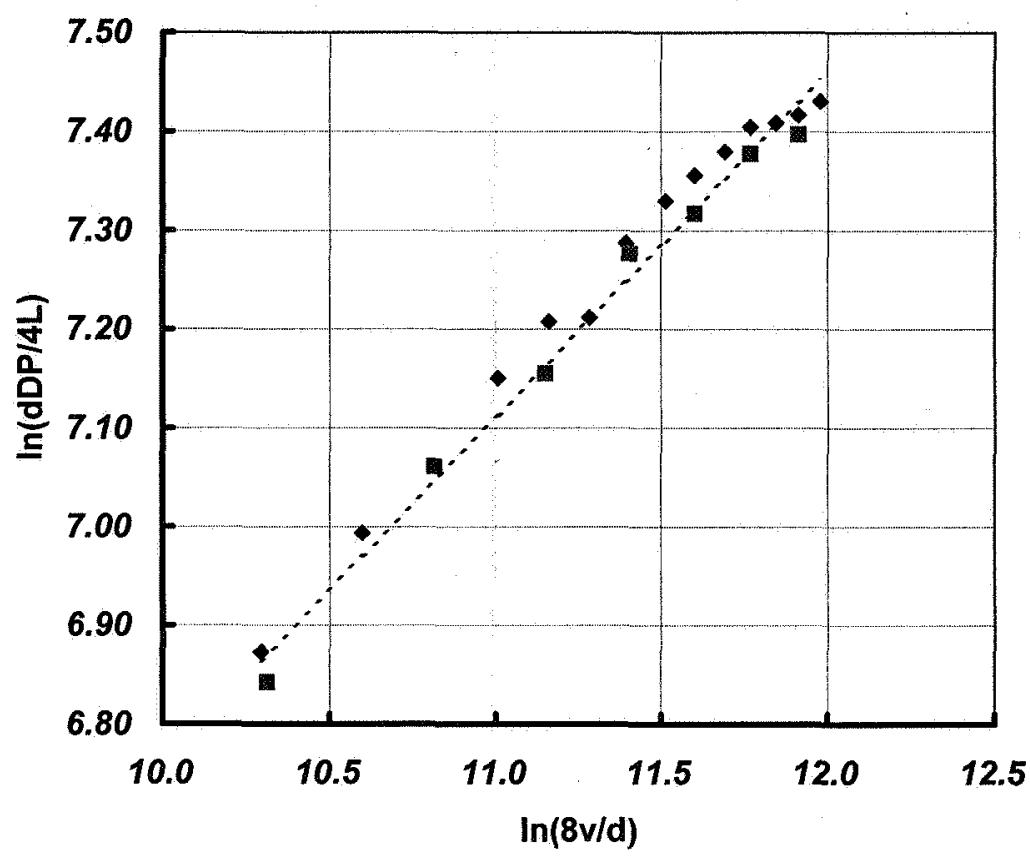

Figure 4 - Logarithmic Representation of the $\tau v s . \gamma$ Data

Thus Eq. 6 is rewritten as

$$
\tau=23.06 \cdot \dot{\gamma}^{0.349}
$$

Eq. 8

These values are consistent with data obtained in previous experiments with a stainless steel tube of 0.084 in diameter and $6 \mathrm{ft}$ length. Additionally, the behavioral index of the silica gel is very similar to the behavior index of buttermilk, as reported by Butler and O'Donnell (1999). The viscosity of the silica gel is therefore

$\mu=23.06 \cdot \dot{\gamma}^{0.349-1}$

Eq. 9

The flow curve of the silica gel is also time dependent. Generally, this dependence is expressed by multiplying Eq. 9 by a time dependent function [Butler, 1999].

$\mu(t, \dot{\gamma})=\lambda(t) \cdot\left[23.06 \cdot \dot{\gamma}^{0.349-1}\right]$

Eq. 10

where, in general $\lambda(t)$ is a function of time. As specified by Butler, (1999) and Eq. 10, the viscosity of the time dependent shear thinning gel can be expressed as a product between a unitless, time dependent function $\lambda(t)$, and a power law, as determined in Eq. 9. The time dependent function, $\lambda(t)$ tracks in fact the evolution of a structural parameter $\lambda$, which is described by a second order kinetic equation derived by Petrellis and Flumerfelt, (1973),

$\frac{d \lambda}{d t}=-k_{1} \cdot\left(\lambda-\lambda_{e}\right)^{2}$

Eq. 11

for $\lambda>\lambda_{\mathrm{e}}$. The structural parameter $\lambda$ ranges from an initial value of unity (at zero time of shear) to an equilibrium value $\left(\lambda_{e}<1\right)$ for $t \rightarrow \infty$ and $k_{1}$ is a rate-constant, which is a function of shear rate. Integrating Eq. 11 we obtain,

$\frac{d\left(\lambda-\lambda_{e}\right)}{\left(\lambda-\lambda_{e}\right)^{2}}=-k_{1} \cdot d t$,

$-\left(\lambda-\lambda_{e}\right)^{-1}=-k_{1} \cdot t-C_{1}$,

Eq. 12

$\lambda(t)=\lambda_{e}+\frac{1}{C_{1}+k_{1} \cdot t}$ 
where $C_{l}$ is a constant of integration. The determination of $\lambda_{\mathrm{e}}, \mathrm{k}_{1}$, and $\mathrm{C}_{1}$ was done by utilizing the already available shear thinning (i.e. power law behavior) data and by employing our Brookfield DV-II+ programmable viscometer with WinGather ${ }^{\mathrm{TM}}$ software to determine the time variation of silica gel viscosity at constant shear rate. A set of five LV-series Brookfield spindles was used to make viscosity measurements over a five order-of-magnitude $\left(10^{-4}-10 \mathrm{~Pa} \cdot \mathrm{sec}\right)$ range.

Extrapolating the measured data, the zero shear viscosity for the water silica gel tested $(12.5 \%$ silica by mass) was $8 \mathrm{~Pa} \cdot \mathrm{sec}$. At the limited shear rate of $7-10 \mathrm{~s}^{-1}$ exerted by the spindle of the viscometer, after 1.5 hours, the viscosity of the gel settled at around $0.2 \mathrm{~Pa} \cdot \mathrm{sec}$ and did not change further to a noticeable degree. Moreover, unlike the gel sheared by the high shear rheometer, this gel recovered its original viscosity once allowed to sit in the test cup for about half an hour.

The estimation of the three unknown constants in Eq. 12 begins by noticing that at the limit of $t \rightarrow \infty$ the structural parameter $\lambda(\mathrm{t}) \rightarrow \lambda_{\mathrm{e}}$. At $\mathrm{t}=4000$ $\mathrm{sec}$, the viscosity of the gel was $0.2 \mathrm{~Pa} \cdot \mathrm{sec}$ and virtually constant. Thus we can write

$$
\lambda_{e} \cdot k \cdot \dot{\gamma}^{n-1}=0.2 P a \cdot \sec \quad \text { Eq. } 13
$$

with $\mathrm{k}=23.06 \mathrm{~Pa} \cdot \mathrm{sec}^{\mathrm{n}}, \mathrm{d} \gamma / \mathrm{dt}=7.3 \mathrm{sec}^{-1}$, and $\mathrm{n}=$ 0.349 . Solving, we obtain $\lambda_{\mathrm{e}}=0.03$. Also, we note that at $\mathrm{t}=0$, the viscosity is $8 \mathrm{~Pa} \cdot \mathrm{sec}$ and $\mathrm{Eq}$. 12 reduces to,

$\lambda=\lambda_{e}+\frac{1}{C_{1}}=0.03+\frac{1}{C_{1}}$,

Eq. 14

and thus

$$
\left(0.03+\frac{1}{C_{1}}\right) \cdot k \cdot \dot{\gamma}^{n-1}=8 P a \cdot \mathrm{sec} \quad \text { Eq. } 15
$$

Solving for $C_{1}$ we obtain $C_{1}=0.81$. To obtain $k_{1}$ we simply remove the shear thinning contribution from the data set. These data is then fitted with a function of the form,

$$
\lambda+0.03=\frac{1}{0.81+k_{1} \cdot t}
$$

from where the value of $\mathrm{k}_{1}=0.022 \mathrm{sec}^{-1}$ is obtained. Summarizing, the silica gel viscosity can thus be written as,

$$
\begin{aligned}
& \mu(t, \dot{\gamma})=\left(0.03+\frac{1}{0.81+0.022 \cdot \sec ^{-1} \cdot t}\right) \cdot \text { Eq. } 17 \\
& \cdot\left(23.06 P a \cdot \sec ^{n}\right) \cdot(\dot{\gamma})^{n-1} .
\end{aligned}
$$

Eq. 17 fits the original data within $\pm 25 \%$ as shown in Figure 5.

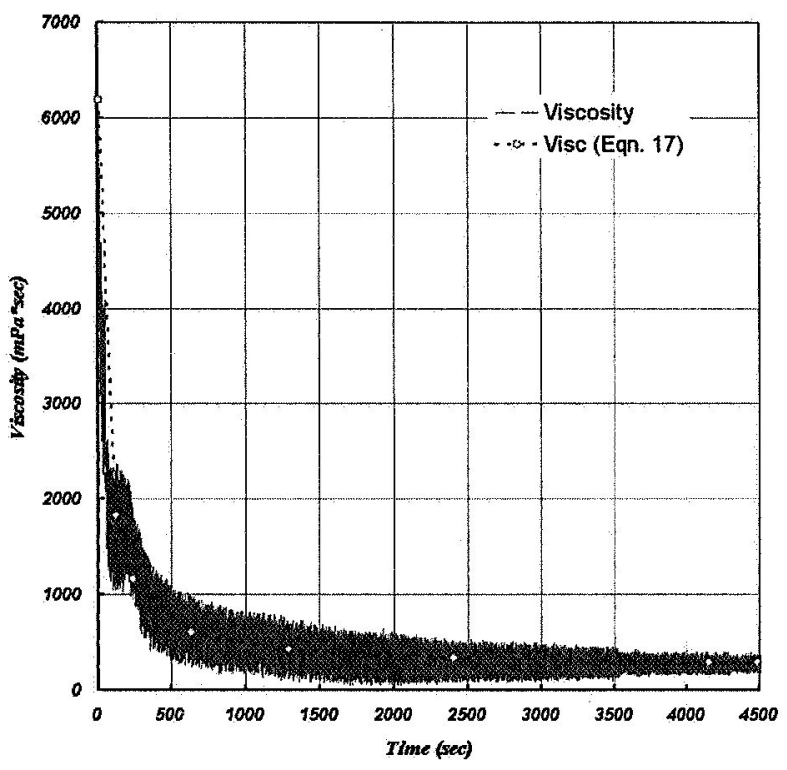

Figure 5 - Original Viscosity Data and Eq. 17 Fit 


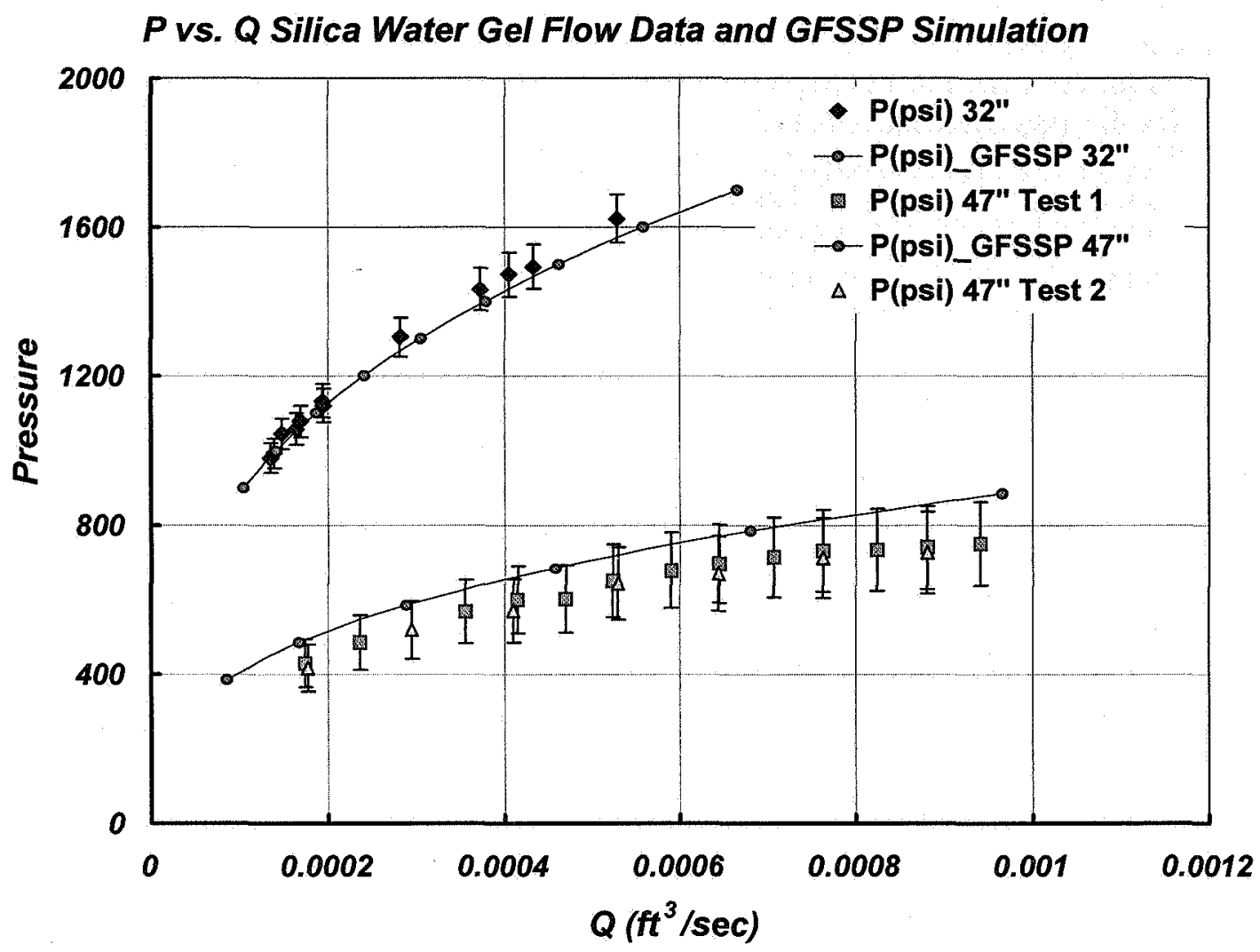

Figure 6 - Experimental Silica Gel Data and GFSSP Simulations

\section{Modeling Results}

The GFSSP simulation included a simple flow circuit similar to the capillary rheometer (see Figure 7).

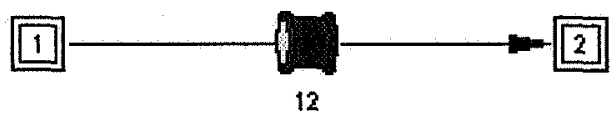

\section{Figure 7 - GFSSP Schematic of Capillary} Rheometer

The boundary nodes specify the tank and discharge pressure and the parameters of the pipe section are set to match the actual pipes tested. The test data for the 0.032 in and 0.047 in diameter tubes, and the corresponding simulated flow curves are shown in Figure 6.
We note from Figure 6 that the 0.032 in tube data is approximated much better $( \pm 4 \%)$ than the 0.047 in tube data $( \pm 15 \%)$. Since the $n$ and $K$ values were calculated with the 0.047 in tube data, and the entrance loss coefficient was calculated from the 0.032 in data, we can conclude that the error in the 0.047 in simulation is due to an overestimation of the pressure loss at the entrance of the 0.047 in tube. Also, we can conclude that the relative insensitivity of the $n$ and $K$ determination to the error made in the evaluation of the entrance effect is due to the fact that the entrance loss manifests itself as a uniform bias of the pressure data, and does not greatly affect the shape of the functional relationship expressed by Eq. 6. These considerations will permit the estimation of the entrance effect from the GFSSP, if some experimental pressure/flowrate data is available. 


\section{Conclusions}

In this study we successfully implemented into GFSSP flow equations for fluids with power law behavior. This required the modification of the sink terms of the momentum equation. This was the first step in the introduction of non-Newtonian, time dependent flow treatment into the GFSSP code. As part of the study, we also developed and experimentally verified the procedure for quantifying the properties of non-Newtonian, time-dependent fluids necessary to define the physical properties of the fluids. Future efforts will be directed towards expanding the code to incorporate treatment of the time dependent behavior.

\section{References}

Butler, F., and O'Donnell, H. J., Modelling the Flow of a Time-Dependent Viscous Product (Cultured Buttermilk) in a Tube Viscometer at $5^{\circ} \mathrm{C}$, Journal of Food Engineering, Vol. 42, pp.199-206, 1999.

Ferguson, J., and Kemblowski, Z., Applied Fluid Rheology, Elsevier Applied Science, London and NY, 1991.

Majumdar, A. K., "Generalized Fluid System Simulation Program (GFSSP) Version 3.0," NASA/MSFC and Sverdrup Technology Inc., MSFC Group, Report No. MG-99-290, November, 1999.

Munson, B. R., Young, D. F., and Okiishi, T. H., Fundamentals of Fluid Mechanics, John Wiley \& Sons, NY, 1990.

Petrellis, N. C. and Flumerfelt, R. W., "Rheological Behavior of Shear Degradable Oils: Kinetic and Equilibrium Properties," Canadian Journal of Chemical Engineering, No. 51, pp. 291301, 1973.

Van Hooser, K., Bailey, J., and Majumdar, A., Numerical Prediction Of Transient Axial Thrust And Internal Flows In A Rocket Engine Turbopump, AIAA 99-2189, $35^{\text {th }}$ AIAA/ASME/SAE/ASEE Joint Propulsion
Conference and Exhibit, 20-24 June 1999, Los Angeles, California. 